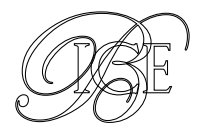

Subdirección General de Estudios y

Evaluación de Instrumentos de Política Comercial*

\section{EL COMERCIO EXTERIOR ANTE LOS RETOS DE 2020}

La pandemia COVID-19 ha alterado profundamente el escenario económico y comercial internacional. La evolución del sector exterior español en 2019 y las tendencias observadas en los años anteriores indican que está en condiciones de contribuir de forma significativa a la superación de los efectos económicos de la pandemia y a la recuperación de la senda de crecimiento económico y de creación de empleo. Las políticas públicas y, en particular, las de apoyo a la internacionalización están llamadas a desempeñar un papel clave en este proceso.

Palabras clave: comercio exterior, internacionalización, exportadores regulares, COVID-19, Brexit, política comercial.

Clasificación JEL: F13, F20, J81.

\section{Introducción}

El 11 de marzo de 2020, el Director General de la Organización Mundial de la Salud (OMS) declaró haber «llegado a la conclusión de que la COVID-19 puede considerarse una pandemia» (OMS, 2020). Esta es la primera pandemia generada por un coronavirus $\mathrm{y}$, como señaló el propio director general, también es la primera pandemia que puede ser controlada.

EI COVID-19 está teniendo profundos efectos desde muchas perspectivas. No solo está generando un profundo sufrimiento humano, sino que produce cambios en las esferas sanitaria, social y política. Desde la perspectiva económica también tendrá repercusiones significativas.

\footnotetext{
* Secretaría de Estado de Comercio.

Versión de marzo de 2020.

DOI: https://doi.org/10.32796/bice.2020.3121.6983
}

Es todavía pronto para valorar el impacto que llegará a tener esta crisis sanitaria en la economía mundial. Las estimaciones iniciales (OCDE, 2020) indican que será de gran magnitud, pero dibujan escenarios muy distintos, en función del éxito que tengan las políticas de contención y de la capacidad de reacción y coordinación de las políticas económicas.

El COVID-19 se añade así a otros factores que ya determinaban un contexto internacional complejo y sujeto a amenazas que podrían condicionar la evolución de la economía y comercio mundiales en los próximos años. En este marco, el objetivo de este artículo es analizar la situación del sector exterior español y evaluar las capacidades con que cuenta para afrontar este incierto escenario.

Para ello, en el apartado 2 se realiza una breve revisión de la situación que presentaban la economía y el comercio internacional al $\triangleright$ 
iniciarse 2020. En el apartado 3 se analizan los resultados del comercio exterior español durante el año 2019, lo que permitirá extraer algunas conclusiones sobre sus capacidades actuales. El apartado 4 se dedica, con una visión más de medio plazo, a estudiar algunas tendencias que han marcado, en las últimas décadas, la evolución del sector exterior. En el apartado 5 se revisa el papel que deberán desempeñar las políticas públicas y, en particular, los instrumentos de apoyo a la internacionalización. El artículo finaliza con un apartado en el que se destacan las conclusiones más relevantes.

\section{La situación de la economía y el comercio internacionales}

La economía mundial presentaba señales de estabilización a finales de 2019. Tras un año de moderación en los ritmos de crecimiento de las principales economías avanzadas, el Fondo Monetario Internacional (FMI), en sus previsiones de enero de 2020 (Cuadro 1), estimaba un crecimiento del PIB mundial en 2019 del $2,9 \%$, siete décimas inferior al del ejercicio anterior (3,6\%), y preveía un crecimiento del 3,3\% en 2020 y del 3,4\% en 2021 (FMI, 2020).

Algunas de las tensiones que habían generado incertidumbre en 2019 parecían atenuarse ante decisiones como la firma, el pasado 15 de enero, del Acuerdo Económico y Comercial entre Estados Unidos de América y la República Popular China (en adelante, Acuerdo EE UU-China) o la ratificación, el 29 de enero, por el Parlamento británico del Acuerdo de Retirada de la Unión Europea (en adelante, Acuerdo de Retirada).

El comercio mundial, estancado en 2019, también parecía estabilizarse a finales del ejercicio (Gráfico 1). Las previsiones, acordes con la mejora del crecimiento económico y la relajación de tensiones geopolíticas y comerciales, apuntaban igualmente a una recuperación de los ritmos de crecimiento del volumen de comercio para 2020 y 2021 (Cuadro 1).

Este escenario de moderada mejora, aunque no exento de riesgos, se ha visto abruptamente alterado por la irrupción del COVID-19. La pandemia, de la que se tuvieron las $\triangleright$

CUADRO 1

ESTIMACIÓN Y PREVISIONES DEL FMI EN ENERO DE 2020

(Tasas de variación interanual)

\begin{tabular}{|c|c|c|c|}
\hline & 2019 & 2020 & 2021 \\
\hline Economías avanzadas & 1,7 & 1,6 & 1,6 \\
\hline Zona euro & 1,2 & 1,3 & 1,4 \\
\hline España & 2,0 & 1,6 & 1,6 \\
\hline Alemania & 0,5 & 1,1 & 1,4 \\
\hline Francia & 1,3 & 1,3 & 1,3 \\
\hline Italia & 0,2 & 0,5 & 0,7 \\
\hline Estados Unidos & 2,3 & 2,0 & 1,7 \\
\hline Japón & 1,0 & 0,7 & 0,5 \\
\hline Reino Unido & 1,3 & 1,4 & 1,5 \\
\hline Economías emergentes y en desarrollo & 3,7 & 4,4 & 4,6 \\
\hline China & 6,1 & 6,0 & 6,8 \\
\hline Comercio mundial (volumen) & 1,0 & 2,9 & 3,7 \\
\hline
\end{tabular}




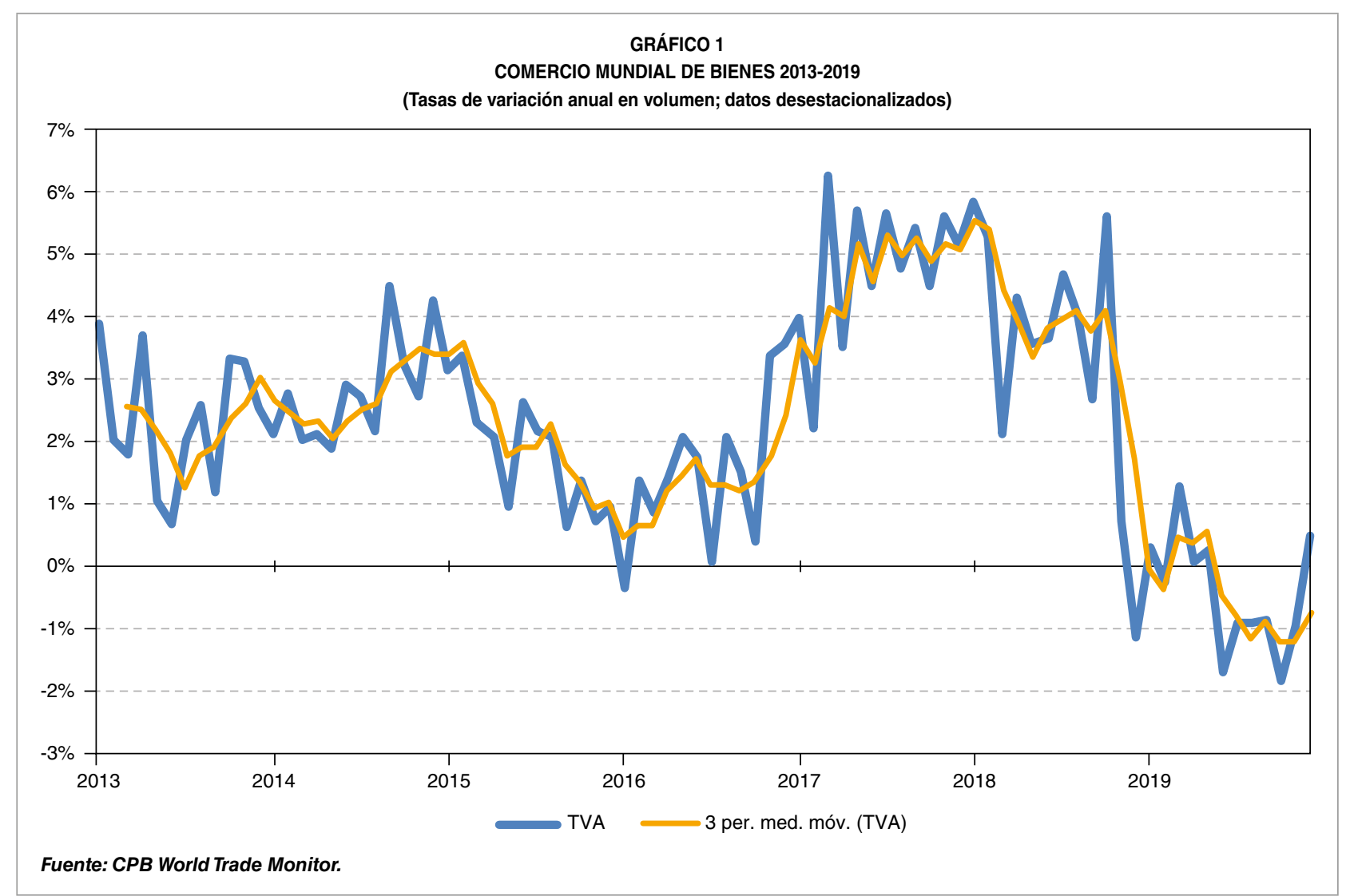

primeras noticias en China a principios del año 2020, afectaba, el 11 de marzo, a 114 países, (OMS, 2020). Tras el notable éxito de las medidas de contención aplicadas en China, Europa se ha convertido, en el momento de escribir este artículo, en su nuevo epicentro.

La experiencia de China muestra que, tanto la epidemia como las medidas adoptadas para contenerla tienen un fuerte impacto económico, cuya duración es, además, muy incierta. La
OCDE señaló, a principios de marzo, que «los indicadores disponibles para febrero apuntan a reducciones significativas de la actividad en China»1 (OCDE, 2020).

La OCDE identifica tres vías por las que estos efectos se produjeron en China y se están produciendo en el conjunto de la economía mundial (Gráfico 2). Así, la pandemia $\triangleright$

\footnotetext{
1 Traducción propia del original en inglés.
}

GRÁFICO 2

CANALES DE TRANSMISIÓN DE LOS EFECTOS ECONÓMICOS DEL COVID-19

Medidas de contención

- Cuarentenas.

- Restricciones a la

movilidad.

- Cierres de espacios

públicos.

Fuente: OECD (2020).

Oferta
- Rierres de industrias.
- Reducciones en la provisión
de servicios.
- Rupturas en las cadenas
de aprovisionamiento.

Demanda
- Pérdida de confianza.
- Viajes de negocios
o turísticos.
- Servicios educativos
y de ocio.


supone un doble shock de oferta y demanda, que a corto plazo se ve reforzado por las medidas de contención que, desde el ámbito sanitario, resulta indispensable adoptar.

Teniendo en cuenta estos canales de transmisión, la OCDE modelizó el posible impacto de la pandemia en dos escenarios.

Un escenario central que, lamentablemente, ha quedado rápidamente obsoleto, partía de la hipótesis de que el brote se centrara básicamente en China. En este escenario la OCDE preveía una reducción de la tasa de crecimiento de la economía mundial, para 2020, de 0,5 puntos porcentuales (Gráfico 3).

En el escenario alternativo, en el que el brote se extiende a las economías desarrolladas y, en particular, a Europa, el crecimiento de la economía mundial se reduciría en 1,5 puntos porcentuales, quedando en aproximadamente la mitad de lo que la propia OCDE había previsto meses antes.

Es pronto para valorar si este segundo escenario puede o no considerarse realista. Día a día, la nueva información sobre la extensión de la pandemia, sobre las medidas de contención que se adoptan en los distintos países y sobre los impactos económicos que comienzan a evidenciarse, puede alterar profundamente las previsiones, tanto en lo que se refiere a su efecto global como a su distribución entre las diferentes regiones del mundo.

Sin embargo, del ejercicio realizado por la OCDE puede concluirse que el impacto será, en todo caso, muy importante y que afectará también notablemente al comercio mundial, cuyo comportamiento en 2020 estará, sin duda, alejado de las moderadamente optimistas previsiones de principios del año.

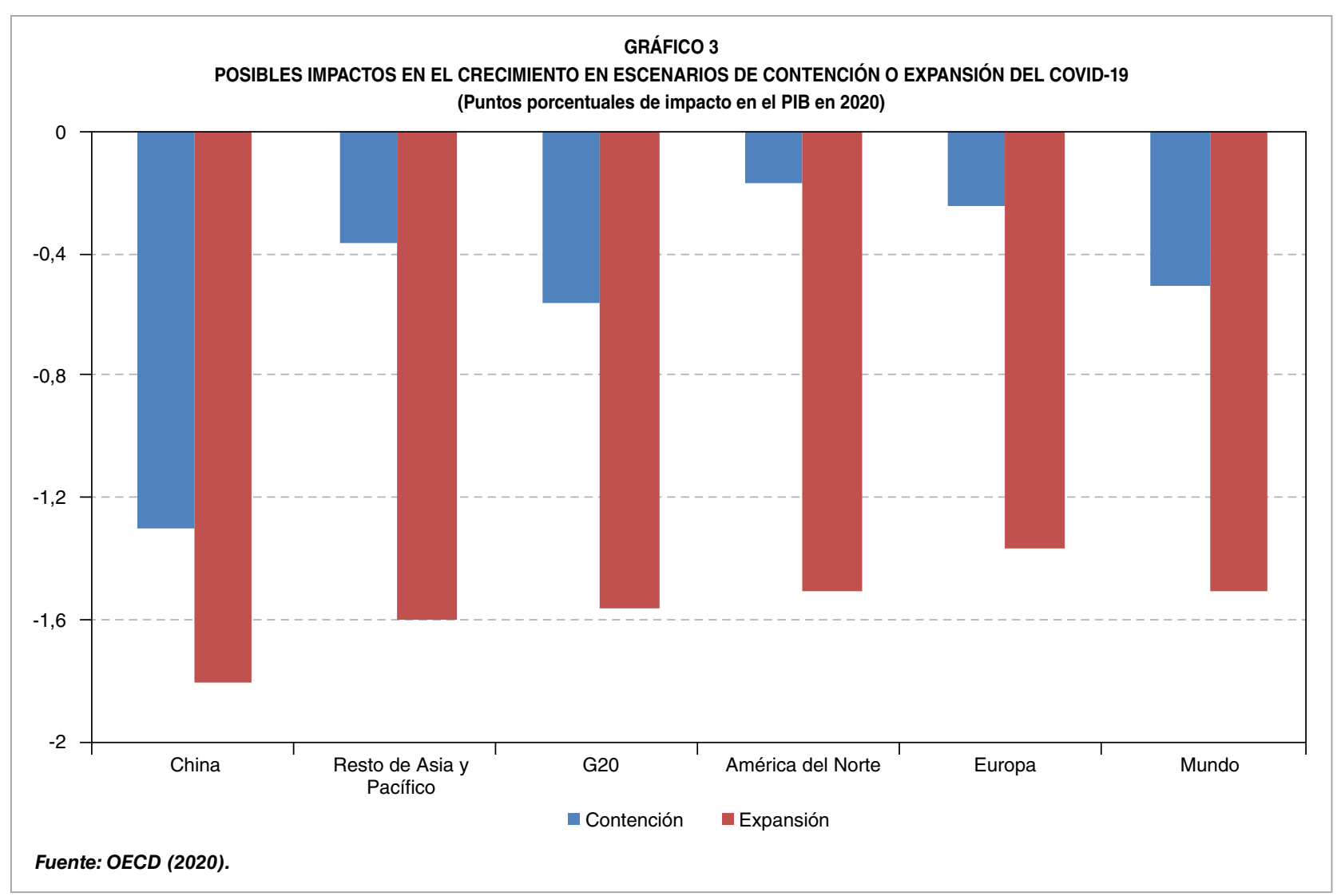


Es preciso recordar, además, que subsisten otros factores de riesgo potencialmente relevantes.

Entre ellos, el resurgimiento de tensiones y medidas proteccionistas en algunos países que afectó al comercio mundial en 2019 y se mantiene como un riesgo significativo para 2020.

Así, el Acuerdo EE UU-China, ya citado, aunque tenga la virtud de reducir las tensiones comerciales entre estas dos grandes economías, podría provocar efectos de desviación de comercio que afectaran negativamente a otros países, entre ellos España. El acuerdo incluye un compromiso de incrementar las importaciones chinas procedentes de EE UU en 200.000 millones de dólares entre 2020 y 2021, en una primera fase, a la que seguiría otra, entre 2022 y 2025, con objetivos de importaciones crecientes aún no determinados.

Los compromisos de incremento de las importaciones exclusivamente de mercancías suponen importes de 63.900 millones de dólares en 2020 y 98.200 millones de dólares en 2021. Estas cifras representan, respectivamente, aumentos del $41,4 \%$ y del $63,6 \%$ sobre las importaciones chinas desde EE UU en 2018. Estos incrementos, de llevarse a efecto, podrían desplazar importaciones chinas de terceros países.

Conviene matizar que parece difícil que China pueda cumplir estos compromisos en los dos próximos años, especialmente tras el impacto económico que está teniendo el COVID-19 y que tendrá efectos limitativos sobre su crecimiento y el de sus importaciones.

Para el caso de España conviene tener presente, además, que en nuestras exportaciones a China destacan los productos agroalimentarios. Existe un escaso solapamiento entre los productos agroalimentarios que exportamos a China y los que exportan EE UU, lo que supondría una limitación a los efectos negativos del Acuerdo EE UU-China sobre nuestro sector exportador.

Adicionalmente, es preciso tener en cuenta la vigencia de los aranceles que, bajo la sección 301 de la Ley de Comercio, EE UU ha impuesto a determinados productos procedentes de la UE. Estos aranceles tienen su origen en las medidas de retorsión aprobadas por la Organización Mundial de Comercio en la resolución de la disputa relativa a los apoyos que, en relación con el proyecto Airbus, la UE y algunos Estados europeos, entre ellos España, otorgan a la industria de grandes aeronaves civiles.

Los aranceles afectan tanto a aeronaves (con un arancel inicial del 10\%, situado actualmente en el $15 \%$ ) como a productos agroalimentarios (25\%) y afectan, en el caso de España, a productos que representaron en 2019 el $4,7 \%$ de nuestras exportaciones a EE UU.

Además, están en marcha las negociaciones entre la UE y Reino Unido sobre las relaciones futuras. El 25 de febrero, el Consejo adoptó una decisión por la que se autoriza la apertura de negociaciones (Consejo de la UE, 2020) a la que se anexaron unas directrices de negociación. Los objetivos de la UE son establecer una asociación económica ambiciosa y equilibrada, que supondría un acuerdo de libre comercio, sin aranceles ni contingentes en materia de mercancías y con mecanismos de cooperación aduanera y convergencia regulatoria, entre otros objetivos. No obstante, Reino Unido parece aspirar a un acuerdo más limitado, que no condicione su capacidad de regulación ni la de llegar a acuerdos comerciales con terceros. Además, declara buscar un acuerdo rápido.

Así, aunque el Acuerdo de Retirada despejó a corto plazo el panorama en torno al Brexit, tiene una naturaleza transitoria y no ha $D$ 
Subdirección General de Estudios y Evaluación de Instrumentos de Política Comercial

desaparecido la amenaza de una salida sin acuerdo o con un acuerdo poco profundo que podría tener también un impacto significativo sobre nuestros flujos comerciales.

\section{El comercio exterior español en 2019}

En este contexto internacional, el sector exterior español deberá contribuir a superar los retos descritos. La apertura al comercio y la inversión son motores clave de la economía española y han estado históricamente asociados a mejoras en el crecimiento y el desarrollo económicos.

La situación de partida del comercio exterior español es relevante para determinar su capacidad de afrontar los desafíos que se plantean para 2020 y los años siguientes.
El ejercicio 2019 fue un año de moderado dinamismo del comercio exterior de mercancías. Las exportaciones crecieron un 1,8\%, alcanzando un valor de 290.089 millones de euros, mientras que las importaciones aumentaron en un $1,0 \%$, en línea con el crecimiento que mostraba la demanda interna.

El déficit comercial se redujo un $5,5 \%$ y la tasa de cobertura se situó en el 90,1\%, 0,9 puntos porcentuales por encima de la registrada en 2018. Esta tasa es similar a la observada en los primeros años de recuperación de la economía española, a pesar del fuerte crecimiento de los últimos años, lo que es indicativo del impulso exportador y de la mejora de la competitividad en la última década (Gráfico 4).

El crecimiento de las exportaciones en España se mantuvo en niveles similares al registrado en la zona euro $(1,9 \%)$, frente a la contracción en EE UU y Japón (con tasas de $\triangleright$

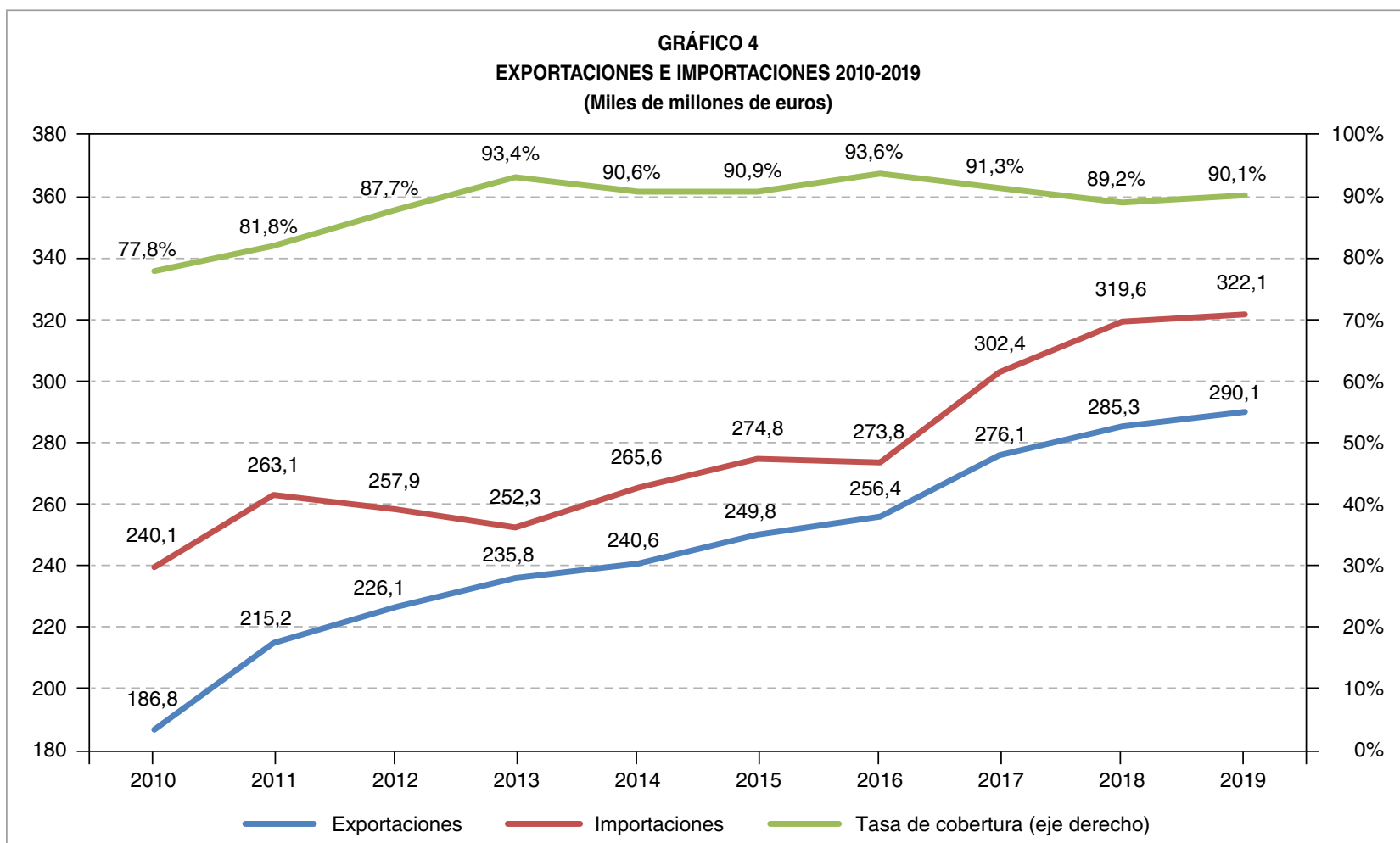

Fuente: SG de Estudios y de Evaluación de Instrumentos de Política Comercial del Ministerio de Industria, Comercio y Turismo, a partir de datos del Departamento de Aduanas e II EE de la Agencia Tributaria. 
variación del $-1,2 \%$ y $-5,5 \%$, respectivamente) y el estancamiento de China, con un aumento del 0,5\% (Cuadro 2).

El dinamismo de las exportaciones de mercancías se apoyó en 2019 en el buen comportamiento de los mercados de algunas regiones avanzadas, como América del Norte o la UE, y de algunas economías emergentes de Asia. Cabe señalar que, a pesar de la incertidumbre generada por el Brexit, las empresas han mantenido y aumentado su presencia en Reino Unido, con un crecimiento de las exportaciones del $3,6 \%$.

En lo que se refiere a las importaciones, destacó el aumento de las procedentes de América del Norte (con un crecimiento del $15,0 \%$ ) y de Asia, excluyendo Oriente Medio $(5,5 \%)$. Las importaciones procedentes de $\square$

CUADRO 2

COMERCIO EXTERIOR EN 2019: DESGLOSE POR ÁREAS GEOGRÁFICAS

\begin{tabular}{|c|c|c|c|c|c|c|}
\hline \multirow{2}{*}{ Área geográfica } & \multicolumn{3}{|c|}{ Exportaciones } & \multicolumn{3}{|c|}{ Importaciones } \\
\hline & $\%$ total & tva $(\%)^{\star}$ & contrib. ** & $\%$ total & tva $(\%)^{*}$ & contrib.** \\
\hline Europa & 71,4 & 1,9 & 1,4 & 59,9 & 1,4 & 0,9 \\
\hline Unión Europea & 65,7 & 2,0 & 1,3 & 53,8 & 1,1 & 0,6 \\
\hline Zona euro & 51,5 & 1,7 & 0,9 & 42,7 & $-0,6$ & $-0,3$ \\
\hline Alemania & 10,7 & 1,0 & 0,1 & 12,4 & $-0,4$ & 0,0 \\
\hline Francia & 15,1 & 2,1 & 0,3 & 10,4 & $-3,1$ & $-0,3$ \\
\hline Italia & 8,0 & 2,1 & 0,2 & 6,4 & $-2,5$ & $-0,2$ \\
\hline Portugal & 7,6 & 3,9 & 0,3 & 3,5 & $-1,2$ & 0,0 \\
\hline Resto UE (*) & 14,3 & 3,2 & 0,5 & 11,1 & 8,2 & 0,9 \\
\hline Reino Unido & 6,8 & 3,6 & 0,2 & 3,7 & 3,1 & 0,1 \\
\hline Resto Europa & 5,7 & 0,8 & 0,0 & 6,0 & 4,8 & 0,3 \\
\hline Turquía & 1,5 & $-9,5$ & $-0,2$ & 2,4 & 6,6 & 0,1 \\
\hline América & 10,8 & 3,2 & 0,3 & 10,6 & 0,7 & 0,1 \\
\hline América del Norte & 5,5 & 9,2 & 0,5 & 5,3 & 15,0 & 0,7 \\
\hline Estados Unidos & 4,7 & 7,4 & 0,3 & 4,8 & 18,1 & 0,7 \\
\hline América Latina & 5,2 & $-1,1$ & $-0,1$ & 4,9 & $-12,7$ & $-0,7$ \\
\hline Brasil & 0,9 & 7,2 & 0,1 & 1,2 & $-21,0$ & $-0,3$ \\
\hline México & 1,5 & $-5,8$ & $-0,1$ & 1,4 & $-3,4$ & $-0,1$ \\
\hline Asia & 9,2 & 2,4 & 0,2 & 20,9 & 1,6 & 0,3 \\
\hline Asia (excepto Oriente Medio) & 6,7 & 7,4 & 0,5 & 18,1 & 5,5 & 1,0 \\
\hline China & 2,3 & 8,4 & 0,2 & 9,1 & 8,3 & 0,7 \\
\hline Japón & 0,9 & 7,8 & 0,1 & 1,4 & 5,4 & 0,1 \\
\hline Oriente Medio & 2,6 & $-8,5$ & $-0,2$ & 2,7 & $-18,7$ & $-0,6$ \\
\hline Arabia Saudí & 0,6 & $-21,4$ & $-0,2$ & 1,3 & $-8,5$ & $-0,1$ \\
\hline África & 6,4 & 0,5 & 0,0 & 8,4 & $-1,9$ & $-0,2$ \\
\hline Marruecos & 2,9 & 3,6 & 0,1 & 2,2 & 4,5 & 0,1 \\
\hline Oceanía & 0,6 & $-2,7$ & 0,0 & 0,2 & $-24,5$ & $-0,1$ \\
\hline Australia & 0,5 & $-2,8$ & 0,0 & 0,2 & $-26,4$ & $-0,1$ \\
\hline Otros $\left({ }^{\star \star}\right)$ & 1,4 & $-10,6$ & $-0,2$ & 0,0 & $-24,1$ & 0,0 \\
\hline Total comercio declarado & 100,0 & 1,8 & 1,8 & 100,0 & 1,0 & 1,0 \\
\hline \multicolumn{7}{|c|}{$\begin{array}{l}\text { * A efectos de cálculo de variación interanual, la comparación se hará con los datos provisionales de } 2018 . \\
{ }^{* *} \text { contrib.: contribución a la tasa de variación anual de las exportaciones/importaciones totales, en puntos porcentuales. }\end{array}$} \\
\hline
\end{tabular}


la UE aumentaron en un $1,4 \%$ y supusieron el $59,9 \%$ del total.

También es destacable el crecimiento de las exportaciones españolas en los mercados de Países con Actuación Sectorial Estratégica (PASE) (Cuadro 3), que son destinos prioritarios de acuerdo con el Plan de Actuación 2019-2020 de la Estrategia de Internacionalización de la Economía Española 2017-2027. Tanto en la vertiente exportadora como en la importadora, los flujos comerciales de España con este grupo de países han crecido por encima del promedio en 2019, con tasas de variación del $4,5 \%$ y 5,9\%, respectivamente. Estos datos indican una mayor presencia española en estos mercados de fuerte crecimiento, donde existen sectores con un elevado potencial de exportación para las empresas españolas.

El comercio exterior de servicios también registró un dinamismo elevado. Con datos acumulados de los tres primeros trimestres de 2019, los ingresos por exportaciones de servicios aumentaron un $6,2 \%$ en tasa interanual, hasta alcanzar los 106.499 millones de euros. Los pagos por importaciones crecieron un $11,5 \%$ y sumaron 57.238 millones de euros. El superávit de la balanza de servicios ascendió a 49.498 millones de euros (Cuadro 4).

Aunque turismo y viajes es la rúbrica que en mayor medida contribuye al saldo superavitario de la balanza de servicios, con una aportación de 38.621 millones de euros en los tres primeros trimestres de 2019, es destacable el fuerte crecimiento de los ingresos de otros servicios. Así, en los tres primeros trimestres del año 2019 los ingresos por exportaciones de este tipo de servicios aumentaron en un 9,6\%, frente al 3,4\% correspondiente a turismo y viajes, continuando la tendencia de fuerte expansión de años anteriores.

En resumen, los resultados que el comercio exterior de bienes y servicios español registró en 2019 permiten afrontar el desafío que se plantea para 2020 desde una posición de partida sólida.

Disponemos de los datos de comercio de bienes en enero, que vienen a confirmar $D$

CUADRO 3

COMERCIO EXTERIOR CON LOS PAÍSES PASE EN 2019

\begin{tabular}{|c|c|c|c|c|c|c|c|}
\hline \multirow{2}{*}{ Países } & \multicolumn{3}{|c|}{ Exportaciones } & \multicolumn{3}{|c|}{ Importaciones } & \multirow{2}{*}{$\begin{array}{c}\text { Saldo } \\
\text { Millones de } €\end{array}$} \\
\hline & Millones de $€$ & $\%$ total & tva (\%) & Millones de $€$ & $\%$ total & tva (\%) & \\
\hline Brasil & $2.598,7$ & 5,0 & 7,2 & $3.818,9$ & 4,5 & $-21,0$ & $-1.220,2$ \\
\hline Canadá & $2.027,7$ & 3,9 & 13,6 & $1.564,4$ & 1,8 & $-10,2$ & 463,3 \\
\hline China & $6.800,8$ & 13,0 & 8,4 & $29.154,5$ & 34,1 & 8,3 & $-22.353,7$ \\
\hline Corea del Sur & $2.257,5$ & 4,3 & 12,2 & $3.118,3$ & 3,6 & $-0,3$ & $-860,9$ \\
\hline Estados Unidos & $13.739,8$ & 26,3 & 7,4 & $15.533,6$ & 18,2 & 18,1 & $-1.793,9$ \\
\hline India & $1.345,4$ & 2,6 & 1,1 & $4.230,9$ & 5,0 & 5,6 & $-2.885,5$ \\
\hline Japón & $2.724,7$ & 5,2 & 7,8 & $4.355,8$ & 5,1 & 5,4 & $-1.631,0$ \\
\hline Marruecos & $8.516,2$ & 16,3 & 3,6 & $6.949,1$ & 8,1 & 4,5 & $1.567,1$ \\
\hline México & $4.295,7$ & 8,2 & $-5,8$ & $4.612,3$ & 5,4 & $-3,4$ & $-316,6$ \\
\hline Rusia & $2.054,1$ & 3,9 & 1,3 & $3.442,0$ & 4,0 & 14,0 & $-1.387,9$ \\
\hline Sudáfrica & $1.448,0$ & 2,8 & 26,0 & $1.100,4$ & 1,3 & $-9,7$ & 347,5 \\
\hline Turquía & $4.465,6$ & 8,5 & $-9,5$ & $7.590,6$ & 8,9 & 6,6 & $-3.125,1$ \\
\hline Com. declarado PASE & $52.274,1$ & 100,0 & 4,5 & $85.470,9$ & 100,0 & 5,9 & $-33.196,8$ \\
\hline
\end{tabular}


CUADRO 4

BALANZA DE PAGOS DE SERVICIOS 2015-2019 (TRIMESTRES 1 A 3)

(Millones de euros y tasas de variación anuales en porcentaje)

\begin{tabular}{|l|c|c|c|c|c|c|c|c|c|c|c|}
\hline \multirow{2}{*}{ Años } & Total & \multicolumn{4}{|c|}{ Turismo y viajes } & \multicolumn{4}{c|}{ Otros servicios } \\
\cline { 2 - 14 } & Saldo & Saldo & Ingresos & Pagos & $\begin{array}{c}\text { Ingr. } \\
\text { (tva \%) }\end{array}$ & $\begin{array}{c}\text { Pagos } \\
\text { (tva \%) }\end{array}$ & Saldo & Ingresos & Pagos & $\begin{array}{c}\text { Ingr. } \\
\text { (tva \%) }\end{array}$ & $\begin{array}{c}\text { Pagos } \\
\text { (tva \%) }\end{array}$ \\
\hline 2015 & 53.440 & 40.585 & 56.310 & 15.724 & 4,5 & 15,8 & 12.855 & 53.208 & 40.354 & 7,0 & 9,7 \\
2016 (p) & 58.696 & 43.354 & 60.351 & 16.998 & 7,2 & 8,1 & 15.344 & 57.568 & 42.224 & 8,2 & 4,6 \\
2017 (p) & 63.708 & 47.071 & 66.681 & 19.610 & 10,5 & 15,4 & 16.637 & 60.790 & 44.152 & 5,6 & 4,6 \\
2018 (p) & 61.948 & 46.331 & 69.023 & 22.692 & 3,5 & 15,7 & 15.617 & 62.890 & 47.274 & 3,5 & 7,1 \\
2018 T1-3 (p) & 49.168 & 38.597 & 55.247 & 16.650 & 2,9 & 16,0 & 10.571 & 45.247 & 34.676 & 3,8 & 7,5 \\
2019 T1-3 (p) & 49.498 & 38.621 & 57.150 & 18.530 & 3,4 & 11,3 & 10.878 & 49.585 & 38.708 & 9,6 & 11,6 \\
\hline
\end{tabular}

Fuente: Banco de España. Balanza de Pagos.

esta favorable situación del sector exterior español al inicio de 2020 (las exportaciones aumentan un 2,7\% interanual). Estos datos de exportaciones no parecen reflejar aún ningún impacto del coronavirus, sin embargo, en las importaciones originarias de China sí se aprecia una caída de determinados productos (equipos informáticos y de telecomunicaciones) probablemente afectados por la interrupción temporal de las cadenas de valor en las que participa China.

\section{Algunas tendencias destacables}

El comportamiento del comercio exterior de bienes en 2019 ha reforzado algunas de las tendencias que han caracterizado su evolución desde la recuperación económica. Estas tendencias vienen a confirmar la potencia y resiliencia de nuestro sector exportador $y$, junto con los resultados de 2019, constituyen una base robusta para afrontar los desafíos que ofrece 2020.

Un primer rasgo es la diversificación geográfica de nuestro comercio exterior, con una menor dependencia de la zona euro. Si en 1999 las exportaciones españolas de bienes a esta zona suponían el 61,9\% del total, en 2019 se limitaron al 51,5\% (Gráfico 5). En contrapartida, han ganado peso las exportaciones a Asia y África. El peso de las exportaciones a América se mantiene prácticamente estable, aunque con cambios en su composición interna, con un aumento de la importancia relativa de América del Norte en detrimento de América Latina.

Similarmente, la concentración de las importaciones españolas de bienes en países de la zona euro ha disminuido en 14,7 puntos porcentuales y han ganado peso otras regiones, como Asia, África o los países europeos no comunitarios.

Estos datos apuntan a una mayor integración de la economía española en la economía global y una mayor presencia de nuestras empresas en mercados de todas las regiones del mundo. Esta integración dota al sector exportador de una mayor robustez frente a shocks adversos concentrados en una región determinada y también de una mayor capacidad para aprovechar impulsos positivos procedentes de algunos mercados.

Un segundo rasgo es la ampliación de la base exportadora. El número de exportadores regulares (operadores que realizaron exportaciones en el año en curso y en los tres ejercicios anteriores) viene aumentando de forma $D$ 
Subdirección General de Estudios y Evaluación de Instrumentos de Política Comercial

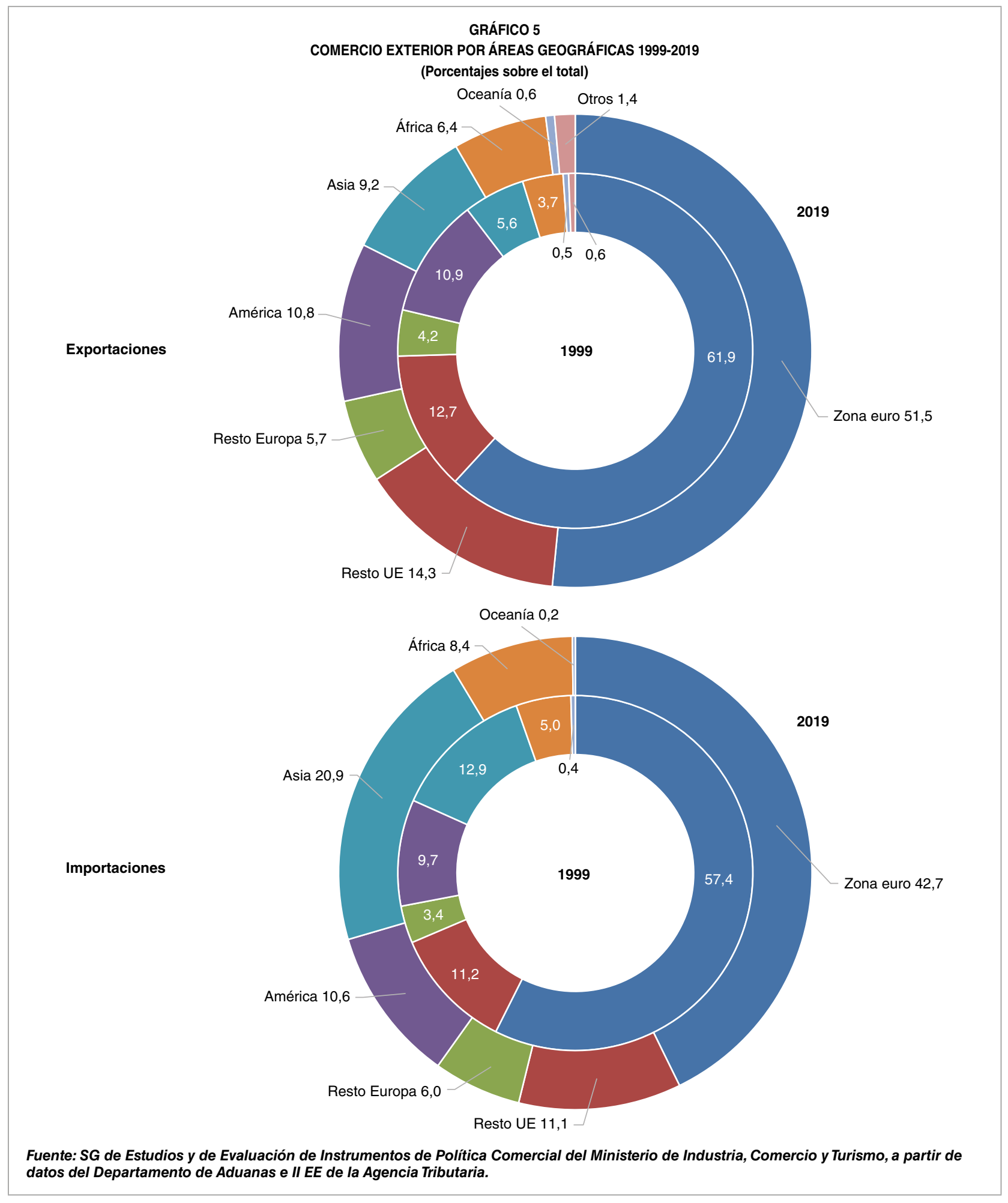

ininterrumpida desde 2011 y se sitúa ya en 52.949 , superando en un $33,6 \%$ el nivel máximo previo a la crisis que se alcanzó en 2008 (Gráfico 6).
Esta base de exportadores regulares cada vez más amplia, que representa en torno al $95,1 \%$ de las exportaciones de mercancías, pone de manifiesto que las empresas $\square$ 


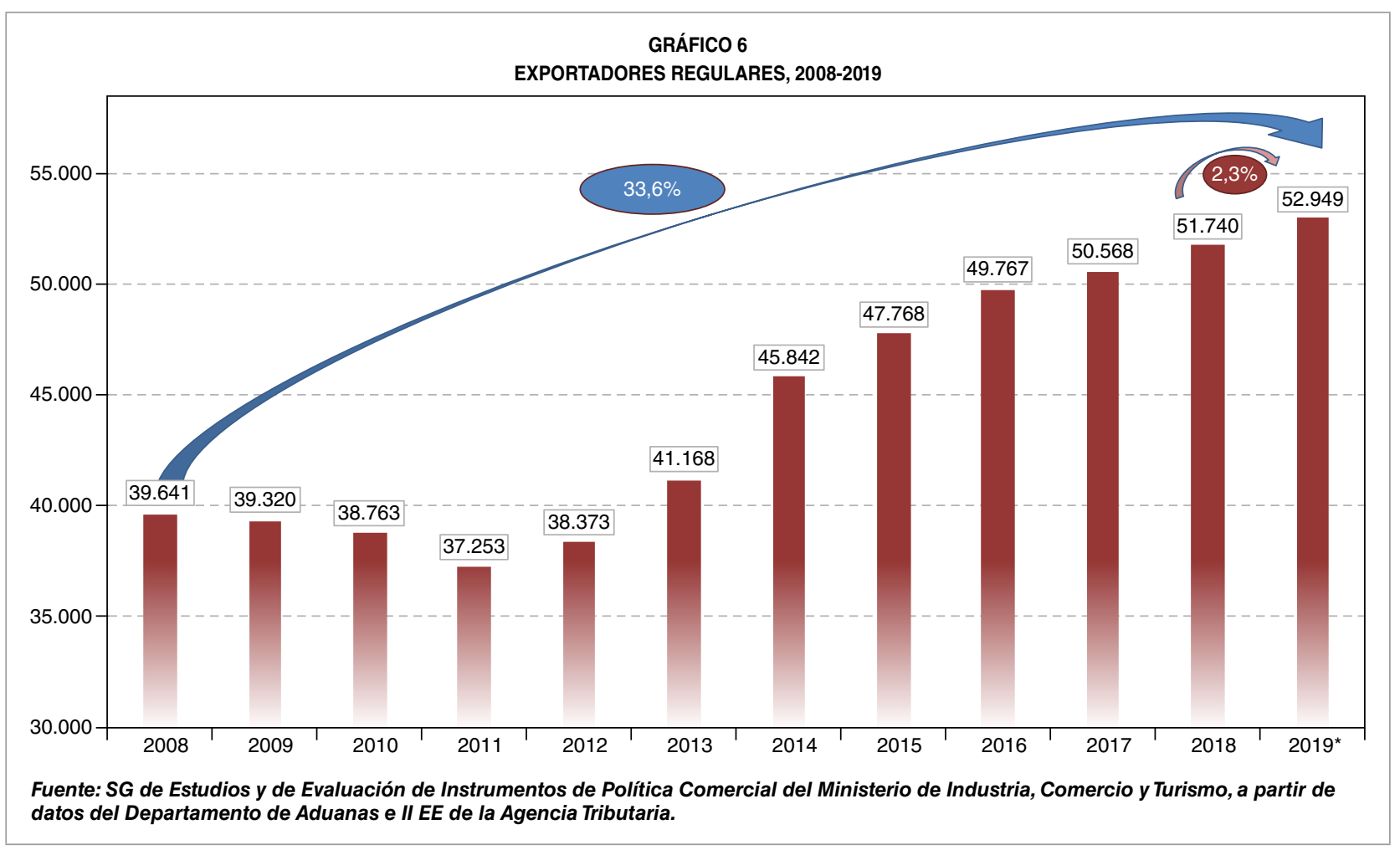

españolas tienen capacidad para mantener y aumentar su presencia en mercados internacionales exigentes y competitivos. Su consolidación, que supone la garantía de poder mantener la capacidad exportadora a largo plazo, es un excelente punto de partida para que el sector exterior continúe contribuyendo positivamente al crecimiento económico.

Un tercer rasgo es la importancia creciente del comercio exterior de servicios y, en particular, de los servicios no turísticos. La terciarización, la globalización y la digitalización favorecen la expansión y el dinamismo del comercio internacional de servicios, que viene creciendo a un ritmo más rápido que el de los bienes. Se espera que esta tendencia continúe en los próximos años. En particular, la digitalización está reduciendo la necesidad de proximidad que condicionaba la prestación de muchos servicios, facilitando la prestación a distancia de servicios financieros, educativos o sanitarios.
Sin olvidar la importancia del turismo para España, destaca el crecimiento de los servicios no turísticos que incluyen sectores como transporte, servicios de telecomunicaciones e informática, servicios financieros y otros servicios empresariales. Entre 1999 y 2018 los ingresos por exportaciones de este tipo de servicios aumentaron en un $308 \%$, frente a un incremento del $124 \%$ de los ingresos por servicios turísticos.

Estos servicios no turísticos muestran un elevado potencial de crecimiento, aunque algunos subsectores, en los que se requiere el desplazamiento de personas a los mercados de destino, pueden verse afectados en el corto plazo por las restricciones a los movimientos derivadas de las medidas de contención del COVID-19. La digitalización de la economía y la conclusión de acuerdos de libre comercio ambiciosos por parte de la Unión Europea en este ámbito, como los firmados con Canadá $\triangleright$ 


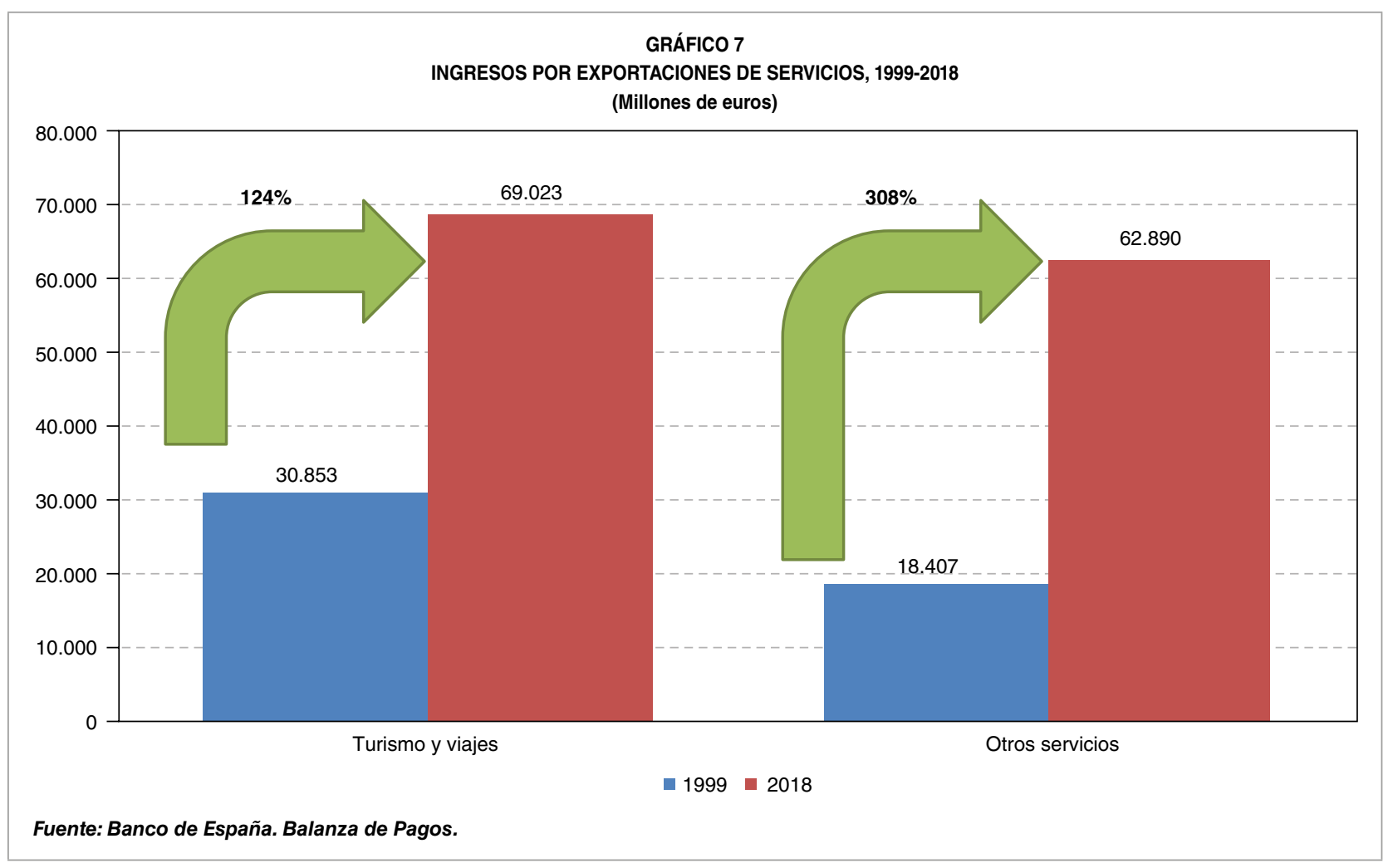

o Japón, son factores que también contribuyen al crecimiento del comercio de estos sectores.

Finalmente, es importante destacar la contribución del sector exterior a un crecimiento más equilibrado. En concreto, 2019 ha sido el octavo año consecutivo con superávit por cuenta corriente y con capacidad de financiación frente al resto del mundo. Además, desde 2014 la economía española ha sido capaz de registrar tasas positivas de crecimiento del PIB al tiempo que mantenía capacidad de financiación frente al resto del mundo, algo inédito en la historia reciente (Gráfico 8 ).

\section{El papel de las políticas públicas y del apoyo a la internacionalización}

Las políticas públicas están llamadas a desempeñar un papel muy relevante no solo en la contención de la pandemia COVID-19 y en la atenuación de sus efectos sociales, sino también para la amortiguación de su impacto económico.

A nivel nacional, se están poniendo en marcha medidas para moderar los efectos sociales y para atenuar también el impacto sobre las empresas. La preservación del tejido empresarial es indispensable para garantizar que las políticas expansivas que puedan aplicarse cuando se hayan levantado las medidas de contención resulten en una pronta recuperación de los niveles de crecimiento económico y de empleo.

Sin embargo, los análisis realizados por la OCDE (Gráfico 9) revelan que para reducir el impacto global será preciso un esfuerzo coordinado de todos los países integrantes del G20. La adopción de medidas de política monetaria y fiscal, combinadas con reformas estructurales, tendrían, si se adoptan por un solo país, un impacto muy limitado. Sólo una acción $\triangleright$ 


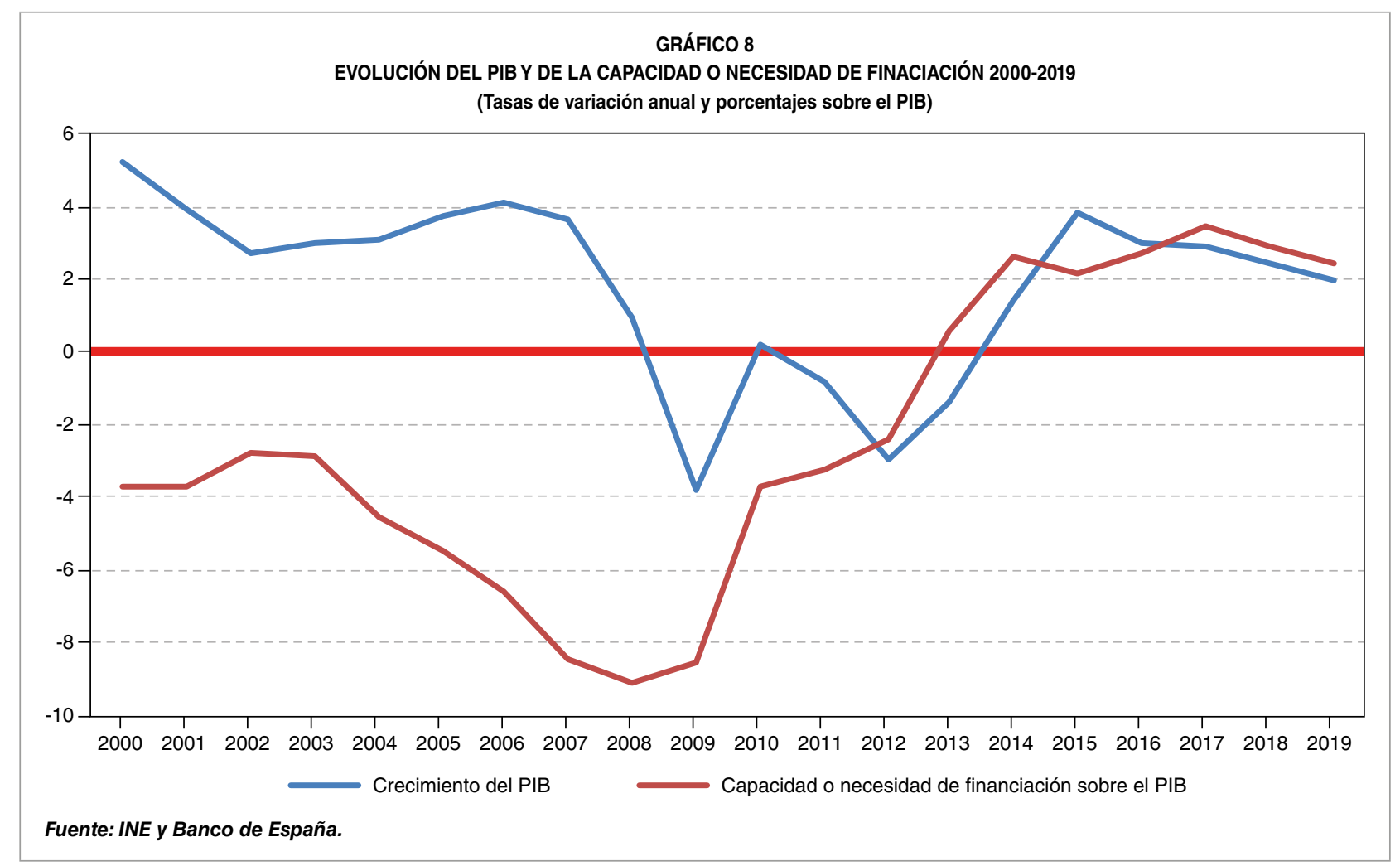

colectiva, que favorezca la recuperación de la confianza en los agentes económicos, podría tener un efecto suficientemente significativo sobre la actividad económica, tanto a corto como a medio y largo plazo. Los principales países del G20 ya han comenzado a anunciar medias expansivas, principalmente en las áreas monetaria y fiscal.

En España, como se ha puesto de manifiesto en los epígrafes anteriores, el sector exterior ha sido clave para el crecimiento en los últimos años. En los próximos, será aún más necesario.

El punto de partida es sólido, como muestran los datos del ejercicio 2019 y las tendencias observadas en las últimas décadas. Pero el entorno internacional va a resultar, con toda probabilidad, más complicado.

Por ello, las políticas públicas de apoyo a la internacionalización deberán ajustarse al nuevo escenario, acompañar a las empresas para afrontar estos retos y contribuir, de esta forma, a recuperar el crecimiento y la creación de empleo.

España dispone de una Estrategia de Internacionalización de la Economía Española 2017-2027 que articula las distintas medidas de apoyo a la internacionalización. Esta estrategia se desarrolla mediante planes bienales, lo que permite adaptarla a la coyuntura cambiante. Su objetivo general es maximizar la contribución del sector exterior al crecimiento y a la creación de empleo, así como la mejora de la competitividad (Subdirección General de Análisis y Estrategia de Internacionalización, 2017).

Entre las medidas ya en ejecución para el bienio 2019-2020, que ahora pueden resultar más relevantes, destacan las siguientes (Subdirección General de Estrategia de Internacionalización, 2019).

En primer lugar, el impulso a la diversificación de mercados y sectores de destino de $D$ 


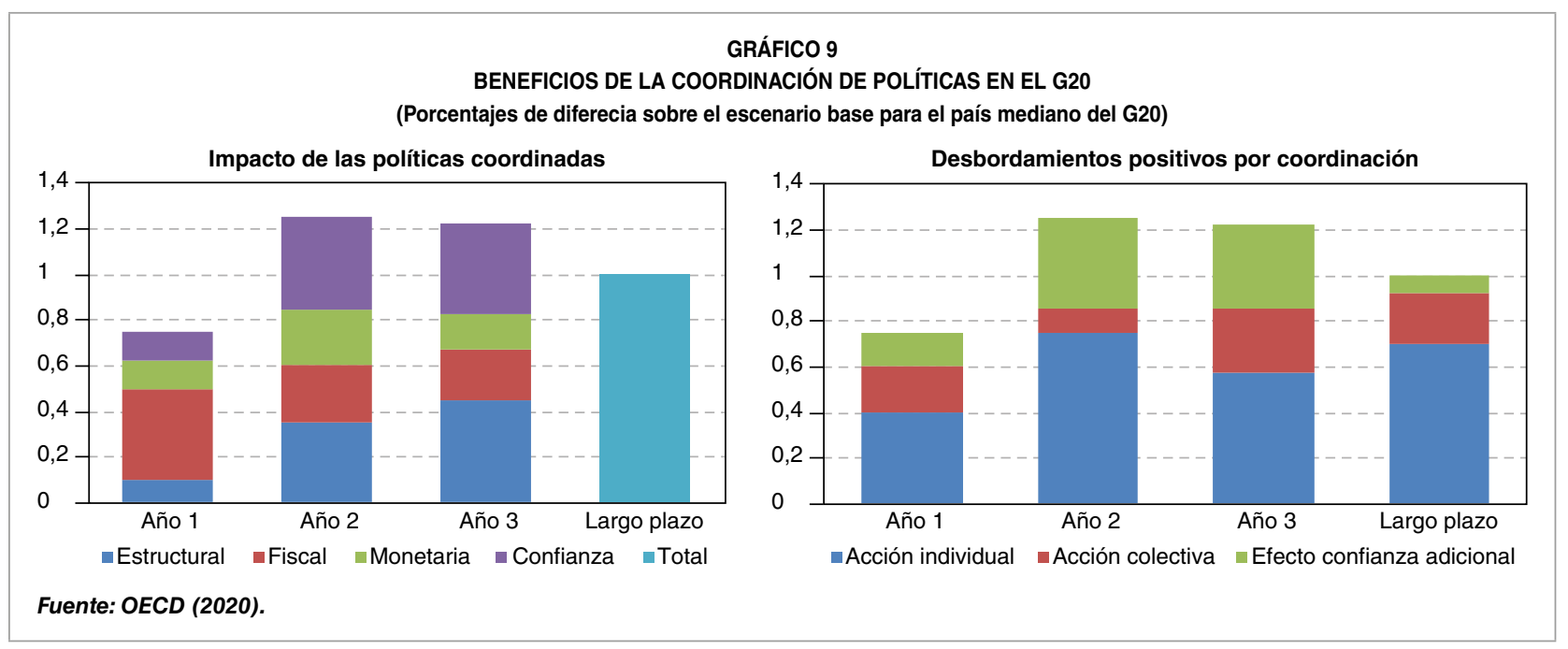

las exportaciones de bienes y servicios españolas a través de los planes PASE. Este ejercicio permite orientar el apoyo público hacia determinados sectores de oportunidad identificados en los distintos países donde las empresas españolas no han alcanzado todo su potencial exportador.

Además, se está elaborando un plan de acción específico para África con los objetivos de analizar las oportunidades de negocio para empresas españolas en este continente, crear programas específicos para apoyar la exportación de productos españoles, identificar nuevos sectores y mercados de oportunidad y reforzar la presencia y apoyo institucional en el continente africano, así como los instrumentos financieros y otros de apoyo a la internacionalización de empresas españolas en dicho continente.

En segundo lugar, la atracción de inversiones exteriores estratégicas en países y sectores prioritarios, que puede contribuir al desempeño de la economía española en 2020 y los años posteriores. Se impulsará un plan de promoción de España como plataforma global de negocios e inversiones, se reforzará el Plan de Sedes, centrado en América Latina, y se potenciarán los programas de apoyo a las inversiones tecnológicas y al emprendimiento en sectores y actividades innovadores.

En tercer lugar, la modernización de los instrumentos de apoyo financiero oficial a las empresas para su internacionalización (FIEM, CESCE por cuenta del Estado, FIEX y FONPYME, CARI): el objetivo es contar con instrumentos más versátiles, adaptados a las necesidades actuales, y al cambiante entorno internacional.

En cuarto lugar, el apoyo a las empresas, fundamentalmente a través de ICEX España Exportación e Inversiones, para incorporar la innovación, la tecnología, la diferenciación de marca, la digitalización y el desarrollo del capital humano en los procesos de internacionalización. Estos apoyos se ajustarán a las necesidades de las empresas prestando servicios de promoción e información integrales, personalizados y con alto valor añadido.

Además, los acuerdos comerciales de la UE con terceros países van a desempeñar un papel clave para abrir nuevas oportunidades de negocio a nuestras empresas. Entre estos acuerdos destaca, por su interés para España, el alcanzado por la UE con Mercosur (Argentina, Brasil, Paraguay y Uruguay). El 28 de junio de 2019 se firmó el acuerdo político entre la UE y Mercosur, que establece las líneas $\triangleright$ 
básicas de un acuerdo comercial ambicioso, equilibrado y de amplio alcance. Desde esta fecha, ambas partes se están centrando en realizar una revisión jurídica del texto acordado para llegar a la versión final del Acuerdo de Asociación y de todos sus aspectos comerciales.

En la negociación de estos acuerdos no deben olvidarse los objetivos de medio y largo plazo, como la promoción de una política comercial progresista e inclusiva, de acuerdo con los Objetivos de Desarrollo Sostenible de la Agenda 2030 y el Acuerdo de París de lucha contra el cambio climático.

\section{Conclusiones}

La extensión de la pandemia COVID-19 ha alterado profundamente las previsiones de evolución de la actividad económica y el comercio internacionales, abriendo paso a un escenario de máxima incertidumbre. Esta disrupción se suma a otros riesgos que ya afectaban al sector exterior, como los conflictos comerciales asociados a tensiones proteccionistas, o las negociaciones sobre las relaciones futuras entre la UE y Reino Unido.

En este marco, los resultados del comercio exterior de España en 2019 y las tendencias de nuestro sector exterior en los últimos años son elementos de apoyo de cara a la recuperación de una senda de crecimiento sostenible y generadora de empleo.

El sector exterior español ha experimentado una profunda transformación en los últimos años apoyada en la mejora de la competitividad, la diversificación de los destinos de nuestra exportación y la ampliación de la base exportadora. Con ello ha contribuido, desde la salida de la crisis económica, a un patrón de crecimiento más equilibrado.
En los próximos años, las políticas públicas y su coordinación internacional jugarán un papel fundamental para superar los efectos ocasionados por la pandemia. En el caso de España, las políticas de apoyo a la internacionalización resultarán también esenciales para que el sector exterior pueda desarrollar todo su potencial y contribuir a la recuperación de un crecimiento sostenido y generador de empleo.

\section{Bibliografía}

Consejo de la UE (2020). Council Decision authorising the opening of negotiations with the United Kingdom of Great Britain and Northern Ireland for a new partnership agreement. https://www.consilium.europa.eu/media/42737/st05870-en20.pdf

Fondo Monetario Internacional (2020). Actualización de las perspectivas de la economía mundial, enero de 2020. https://www.imf.org/es/Publications/WEO/Issues/2020/01/20/weo-updatejanuary2020

OECD (2020). OECD Economic Outlook, Interim Report March 2020, OECD Publishing. https:// doi.org/10.1787/7969896b-en

Organización Mundial de la Salud (2019). Alocución de apertura del Director General de la OMS en la rueda de prensa sobre la COVID-19 celebrada el 11 de marzo de 2020. https://www.who.int/ es/dg/speeches/detail/who-director-general-sopening-remarks-at-the-media-briefing-on-covid-19---11-march-2020

Subdirección General de Análisis y Estrategia de Internacionalización (2017). La estrategia de internacionalización de la economía española 2017-2027. Boletín Económico de ICE, (3093). https://doi.org/10.32796/bice.2017.3093.5674

Subdirección General de Estrategia de Internacionalización (2019). El Plan de Acción para la Internacionalización de la Economía Española 2019-2020. Boletín Económico de ICE (3111). https://doi.org/10.32796/bice.2019.3111.6827 